\title{
"An Alternate Approach to Find an Optimal Solution of a Transportation Problem."
}

\author{
Reena .G.Patel ${ }^{1}$, Dr.P.H.Bhathawala ${ }^{2}$, Dr. Bhavin .S.Patel ${ }^{3}$ \\ $\left({ }^{1}\right.$ Assistant Professor, Department of Applied Science and Humanities, S.P.C.E, Visnagar,North Gujarat). \\ $\left({ }^{2}\right.$ Professor and Head (Retired) Veer Narmad South Gujarat University, Surat). \\ ${ }_{(}^{3}$ Assistant Professor, Department of Applied Science and Humanities, S.P.C.E, Visnagar, North Gujarat)
}

\begin{abstract}
The Transportation Problem is the special class of Linear Programming Problem. It arises when the situation in which a commodity is shipped from sources to destinations. The main object is to determine the amounts shipped from each sources to each destinations which minimize the total shipping cost while satisfying both supply criteria and demand requirements. In this paper, we are giving the idea about to finding the Initial Basic Feasible solution as well as the optimal solution or near to the optimal solution of a Transportation problem using the method known as "An Alternate Approach to find an optimal Solution of a Transportation Problem". An Algorithm provided here, concentrate at unoccupied cells and proceeds further. Also, the numerical examples are provided to explain the proposed algorithm. However, the above method gives a step by step development of the solution procedure for finding an optimal solution.
\end{abstract}

Keywords: Transportation problem, Initial basic feasible solution, degenerate solution, Optimality.

\section{Introduction}

Transportation problem is the most useful special class of Linear programming problem, which is to be used for different sources of supply to different destination of demand in such a way that the total transportation cost should be minimized. Usually the Initial Basic Feasible solution for any transportation problem is obtained by, North-west corner method (NWCM), Least-cost Method (LCM), Vogel's Approximation Method (VAM). Then after, the optimality of the given transportation problem can be checked by MODI. Since Last few years, many manufacturers had used the optimization technique most frequently in Linear Programming Problem, to solve the real world problems. For that, it is crucial to introduce new approaches that allow the model to fit in to the real world as much as possible.

Transportation problem was first discovered by F.L.Hitchcock [1] in his paper "The Distribution of a product from several sources to numerous Localities" and then after its presenting by T.C.Koopmans[2] in his historic paper "Optimum Utilization of the Transportation system." These two contributions helped in the development of transportation methods which involve a number of shipping sources and a number of destinations.Lai and Huang [1992] considered the situations where all parameters are in fuzzy number. Lai and Huang, 1992 assume that the parameters have a triangular possibility distribution. Bazarra, Jarvis and Sherali [1990] define linear programming problems with fuzzy numbers and simplex method is used for finding an optimal solution of the fuzzy transportation problem. Swarup, Gupta and Mohan [2006] explain the method to obtain sensitivity analysis or post optimality analysis of the different parameters in the linear programming problems.This paper presents a new simple approach to find the optimal solution of a Transportation problem. Proposed algorithm gives the idea about the flow of step by step procedure. Also, the numerical examples are provided here for the better explanation of Algorithm.

\section{Mathematical Beyground}

Let us consider the standard balanced transportation problem with $\mathrm{m}$ sources $A_{i}$ (with supplies $a_{i}$ ), i $\in$ $I=\{1,2,3 \ldots \ldots . . \mathrm{m}\}$ and $\mathrm{n}$ destinations $B_{j}$ (with demands $b_{j}$ ), $\mathrm{j} \in J=\{1,2,3, \ldots \ldots, \mathrm{n}\}$.

If $X_{i j}=$ the number of load units moving from $A_{i}$ to $B_{j}$, the feasible solution (x) and set of feasible solutions (X) is:

$\mathrm{X}=\left\{\mathrm{x} / \sum_{j \in J} X_{i j}=a_{i}, \forall \mathrm{i} \in \mathrm{I} ; \equiv \sum_{\mathrm{i} \in \mathrm{I}} \mathrm{X}_{\mathrm{ij}}=b_{j}, \forall j \in J ; \mathrm{X}_{\mathrm{ij}} \geq 0 \forall(\mathrm{i}, \mathrm{j}) ; \sum a_{i}=\sum b_{j}\right\}$.

Mathematically the problem can be stated as minimize $z=\sum_{i=1}^{m} \sum_{j=1}^{n} C_{i j} x_{i j}$ subject to

$\sum_{j=1}^{n} x_{i j}=a_{i} ;$ for $i=1,2, \ldots . . m$ (supply constraints) And

$\sum_{i=1}^{n} x_{i j}=b_{j}$ For $\mathrm{j}=1,2 \ldots \mathrm{n}$ (demand constraints) $\mathrm{X}_{\mathrm{ij}} \geq 0$ for all $\mathrm{i} \& j$.

A transportation problem is said to be balanced if the total supply from all sources equals to the total demands in all destinations i.e. $\sum_{i=1}^{m} a_{i}=\sum_{j=1}^{n} b_{j}$, otherwise it is called the unbalanced transportation problem. 
Transportation Problem

\begin{tabular}{|c|c|c|c|c|c|}
\hline \multirow[t]{2}{*}{ Origins (i) } & \multicolumn{4}{|l|}{ Destinations (j) } & \multirow{2}{*}{ Supply $\left(a_{i}\right)$} \\
\hline & 1 & 2 & ............... & $\mathrm{n}$ & \\
\hline 1 & \begin{tabular}{|l|}
$X_{11}$ \\
\end{tabular} & \begin{tabular}{|l|}
$X_{12}$ \\
\end{tabular} & ……............ & \begin{tabular}{|l|}
$X_{1 n}$ \\
\end{tabular} & $a_{1}$ \\
\hline 2 & $X_{21}$ & $X_{22}$ & $\ldots \ldots \ldots \ldots .$. & $X_{2 n}$ & $a_{2}$ \\
\hline 3 & \begin{tabular}{|l|}
$X_{31}$ \\
\end{tabular} & $X_{32}$ & & $X_{3 n}$ & $a_{3}$ \\
\hline 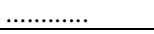 & .......... & $\ldots \ldots \ldots \ldots$ & ........... & ........... & ........... \\
\hline $\mathrm{M}$ & $X_{m 1} C_{m 1}$ & $X_{m 2}$ & ……............ & $X_{m n}$ & $a_{m}$ \\
\hline Demand $\left(b_{j}\right)$ & $b_{1}$ & $b_{2}$ & ……... & $b_{n}$ & $\sum a_{i}=\sum b_{j}$ \\
\hline
\end{tabular}

\section{Proposed Algorithm:}

Step: 1 Construct the transportation matrix from the given transportation problem.

Step: 2Find an IBFS using any one of the method as NWCM, LCM, VAM.

Step:3Then find the minimum cost only from unoccupied cells (non basic variables) from the matrix.

Step:4 Assign $+\theta$ to the minimum unoccupied cell and start to make a loop with occupied cells. Then find $\theta=\min (-\theta)$ and add that $\min (-\theta)$ value at $+\theta$ and subtract that $\min (-\theta)$ value from $-\theta$. Then find the cost of the matrix.

Step: 5continue this process unless and until the optimality has been checked by the any one of the following criteria.

(1) Exactly min least cost as per the condition $\mathrm{m}+\mathrm{n}-3$ in consecutive manner.

(2) If most min cost is skipped, and then also from the next consecutive least min cost should follow the condition $m+n-3$ in consecutive manner also.

(3) If two most min cost are skipped, and then also from the next consecutive least minimum cost should follow the condition $m+n-3$.

(4) If any one of the two most minimum costs are skipped then also number of allocations should follow the condition $m+n-3$.

(5) If any cost is zero in transportation matrix then also as per the criteria no-3 should satisfied.

Step: 6: Now the total minimum cost is calculated as sum of the product of cost and corresponding allocated value of Supply/demand. I.e. Total cost $=\sum_{i=1}^{m} \sum_{j=1}^{n} C_{i j} x_{i j}$

\section{Numerical Examples(Proposed Method):}

1) Consider the following Cost minimizing Transportation problem:

\begin{tabular}{|l|l|l|l|l|l|l|}
\hline & \multicolumn{1}{|c|}{$\boldsymbol{D}_{\mathbf{1}}$} & \multicolumn{1}{c|}{$\boldsymbol{D}_{\mathbf{2}}$} & \multicolumn{1}{c|}{$\boldsymbol{D}_{\mathbf{3}}$} & \multicolumn{1}{c|}{$\boldsymbol{D}_{\mathbf{4}}$} & \multicolumn{1}{c|}{$\boldsymbol{D}_{\mathbf{5}}$} & Supply \\
\hline $\boldsymbol{S}_{\mathbf{1}}$ & 3 & 4 & 6 & 8 & 9 & $\mathbf{2 0}$ \\
\hline $\boldsymbol{S}_{\mathbf{2}}$ & 2 & 10 & 1 & 5 & 8 & $\mathbf{3 0}$ \\
\hline $\boldsymbol{S}_{\mathbf{3}}$ & 7 & 11 & 20 & 40 & 3 & $\mathbf{1 5}$ \\
\hline $\boldsymbol{S}_{\mathbf{4}}$ & 2 & 1 & 9 & 14 & 16 & $\mathbf{1 3}$ \\
\hline Demand & $\mathbf{4 0}$ & $\mathbf{6}$ & $\mathbf{8}$ & $\mathbf{1 8}$ & $\mathbf{6}$ & Total=78 \\
\hline
\end{tabular}

After Applying the Least Cost Method, for Initial Basic Feasible Solution, the Allocations are as follows.

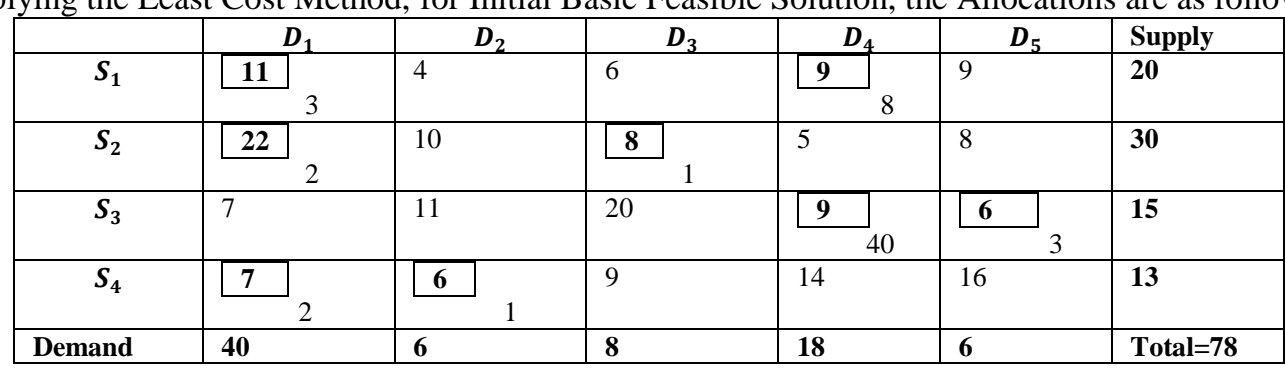

The minimum cost using LCM is obtained as follows,

Min Cost: $(11 * 3)+(9 * 8)+(22 * 2)+(8 * 1)+(9 * 40)+(6 * 3)+(7 * 2)+(6 * 1)$

$$
=555
$$


Now by applying the proposed Method, allocations are obtained as follows,

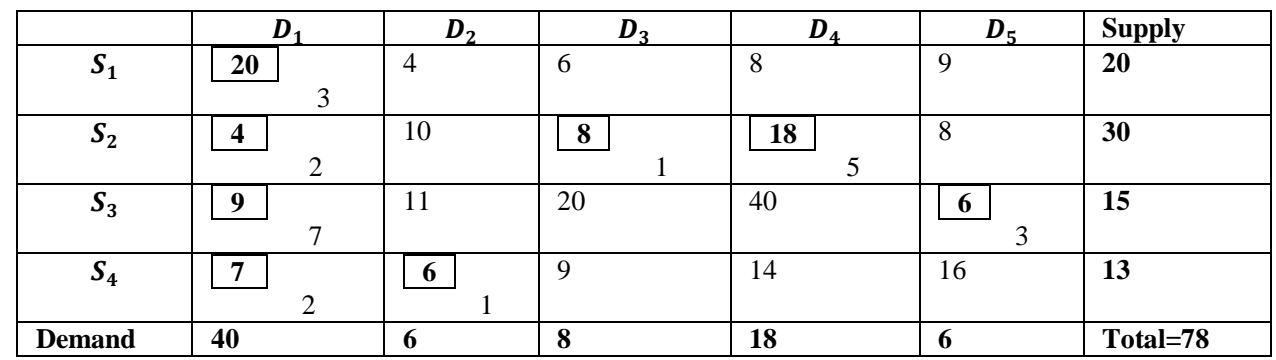

Total Cost obtained by proposed method is as follows,

Total Minimum Cost $=(20 * 3)+(4 * 2)+(8 * 1)+(18 * 5)+(9 * 7)+(6 * 3)+(7 * 2)+(6 * 1)$

$$
=267
$$

2) Consider the following Cost minimizing Transportation problem:

\begin{tabular}{|l|l|l|l|l|l|}
\hline & \multicolumn{1}{|c|}{$\boldsymbol{D}_{\mathbf{1}}$} & \multicolumn{1}{c|}{$\boldsymbol{D}_{\mathbf{2}}$} & $\boldsymbol{D}_{\mathbf{3}}$ & \multicolumn{1}{c|}{$\boldsymbol{D}_{\mathbf{4}}$} & Supply \\
\hline $\boldsymbol{S}_{\mathbf{1}}$ & 19 & 30 & 50 & 10 & $\mathbf{7}$ \\
\hline $\boldsymbol{S}_{\mathbf{2}}$ & 70 & 30 & 40 & 60 & $\mathbf{9}$ \\
\hline $\boldsymbol{S}_{\mathbf{3}}$ & 40 & 8 & 70 & 20 & $\mathbf{1 8}$ \\
\hline Demand & $\mathbf{5}$ & $\mathbf{8}$ & $\mathbf{7}$ & $\mathbf{1 4}$ & Total=34 \\
\hline
\end{tabular}

After Applying the North West Corner Rule, for Initial Basic Feasible Solution, the Allocations are as follows.

\begin{tabular}{|c|c|c|c|c|c|}
\hline 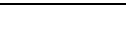 & $D_{1}$ & $D_{2}$ & $D_{3}$ & $D_{4}$ & Supply \\
\hline$S_{1}$ & \begin{tabular}{l|l}
5 & \\
\end{tabular} & 2 & 50 & 10 & 7 \\
\hline$S_{2}$ & 70 & 30 & 40 & 60 & 9 \\
\hline$S_{3}$ & 40 & 8 & 70 & 14 & 18 \\
\hline Demand & 5 & 8 & 7 & 14 & Total $=34$ \\
\hline
\end{tabular}

The minimum cost using NWCM is obtained as follows,

Min Cost: $(5 * 19)+(2 * 30)+(6 * 30)+(3 * 40)+(4 * 70)+(14 * 20)$

$$
=\mathbf{1 0 1 5}
$$

Now by applying the proposed Method, allocations are obtained as follows,

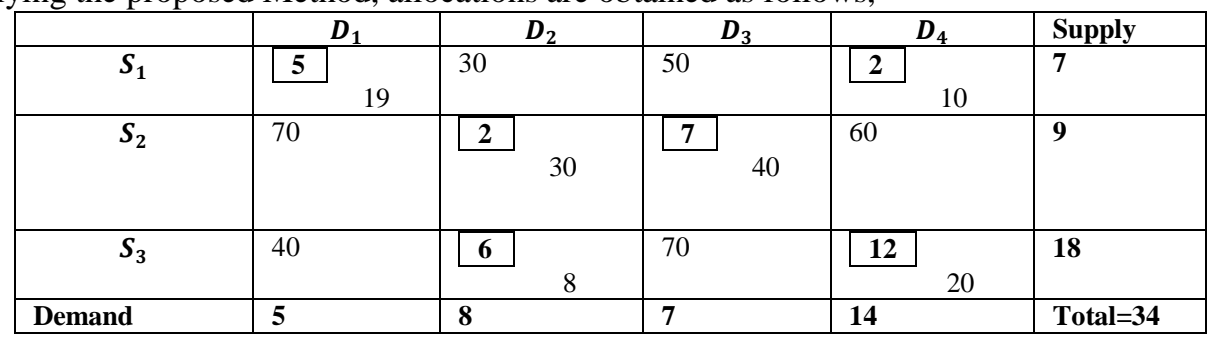

Total Cost obtained by proposed method is as follows,

Total Minimum Cost $=(19 * 5)+(2 * 10)+(2 * 30)+(7 * 40)+(6 * 8)+(12 * 20)$

$$
=743
$$

3) Consider the following Cost minimizing Transportation problem:

\begin{tabular}{|c|l|l|l|l|}
\hline & \multicolumn{1}{|c|}{$\boldsymbol{D}_{\mathbf{1}}$} & \multicolumn{1}{c|}{$\boldsymbol{D}_{\mathbf{2}}$} & \multicolumn{1}{c|}{$\boldsymbol{D}_{\mathbf{3}}$} & Supply \\
\hline $\boldsymbol{S}_{\mathbf{1}}$ & 6 & 4 & 1 & $\mathbf{5 0}$ \\
\hline $\boldsymbol{S}_{\mathbf{2}}$ & 3 & 8 & 7 & $\mathbf{4 0}$ \\
\hline $\boldsymbol{S}_{\mathbf{3}}$ & 4 & 4 & 2 & $\mathbf{6 0}$ \\
\hline Demand & $\mathbf{2 0}$ & $\mathbf{9 5}$ & $\mathbf{3 5}$ & Total=150 \\
\hline
\end{tabular}


After Applying the North West Corner Rule, for Initial Basic Feasible Solution, the Allocations are as follows.

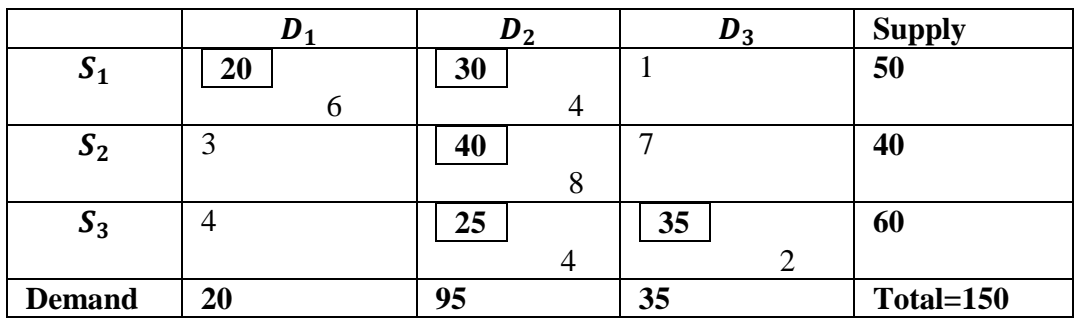

The minimum cost using NWCM is obtained as follows,

Min Cost $:(20 * 6)+(30 * 4)+(40 * 8)+(25 * 4)+(35 * 2)$

$$
=730
$$

Now by applying the proposed Method, allocations are obtained as follows,

\begin{tabular}{|c|c|c|c|c|}
\hline 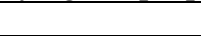 & $D_{1}$ & $D_{2}$ & $D_{3}$ & Supply \\
\hline$S_{1}$ & 6 & \begin{tabular}{l|}
15 \\
\end{tabular} & \begin{tabular}{|l|}
35 \\
\end{tabular} & 50 \\
\hline$S_{2}$ & 20 & 20 & 7 & 40 \\
\hline$S_{3}$ & 4 & 60 & 2 & 60 \\
\hline Demand & 20 & 95 & 35 & Total $=150$ \\
\hline
\end{tabular}

Total Cost obtained by proposed method is as follows,

Total Minimum Cost $=(15 * 4)+(35 * 1)+(20 * 3)+(20 * 8)+(60 * 4)$

$$
\mathbf{5 5 5}
$$

4) Consider the following Cost minimizing Transportation problem:

\begin{tabular}{|c|l|l|l|l|l|}
\hline & \multicolumn{1}{|c|}{$\boldsymbol{D}_{\mathbf{1}}$} & \multicolumn{1}{c|}{$\boldsymbol{D}_{\mathbf{2}}$} & \multicolumn{1}{c|}{$\boldsymbol{D}_{\mathbf{3}}$} & \multicolumn{1}{c|}{$\boldsymbol{D}_{\mathbf{4}}$} & Supply \\
\hline $\boldsymbol{S}_{\mathbf{1}}$ & 4 & 3 & 0 & 5 & $\mathbf{8}$ \\
\hline $\boldsymbol{S}_{\mathbf{2}}$ & 9 & 7 & 3 & 2 & $\mathbf{2}$ \\
\hline $\boldsymbol{S}_{\mathbf{3}}$ & 1 & 5 & 4 & 3 & $\mathbf{9}$ \\
\hline $\boldsymbol{S}_{\mathbf{4}}$ & 6 & 8 & 24 & 16 & $\mathbf{2}$ \\
\hline Demand & $\mathbf{5}$ & $\mathbf{7}$ & $\mathbf{6}$ & $\mathbf{3}$ & Total=21 \\
\hline
\end{tabular}

\begin{tabular}{|c|c|c|c|c|c|}
\hline . & $D_{1}$ & $D_{2}$ & $D_{3}$ & $D_{4}$ & Supply \\
\hline$S_{1}$ & \begin{tabular}{|l|l|}
5 & \\
\end{tabular} & 3 & 0 & 5 & 8 \\
\hline$S_{2}$ & 9 & 2 & 3 & 2 & 2 \\
\hline$S_{3}$ & 1 & 2 & 6 & 1 & 9 \\
\hline$S_{4}$ & 6 & 8 & 24 & 2 & 2 \\
\hline Demand & 5 & 7 & 6 & 3 & Total $=2$ \\
\hline
\end{tabular}

After Applying the North West Corner Rule, for Initial Basic Feasible Solution, the Allocations are as follows.

The minimum cost using NWCM is obtained as follows,

Min Cost: $(5 * 4)+(3 * 3)+(2 * 7)+(2 * 5)+(6 * 4)+(1 * 3)+(2 * 16)$

$$
=112
$$

\begin{tabular}{|c|c|c|c|c|c|}
\hline 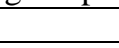 & $D_{1}$ & $D_{2}$ & $D_{3}$ & $D_{4}$ & Supply \\
\hline$S_{1}$ & 2 & 6 & 0 & 5 & 8 \\
\hline$S_{2}$ & 9 & 1 & 3 & 1 & 2 \\
\hline$S_{3}$ & \begin{tabular}{|l|}
3 \\
\end{tabular} & 5 & 6 & 3 & 9 \\
\hline$S_{4}$ & 6 & 8 & 24 & 2 & 2 \\
\hline Demand & 5 & 7 & 6 & 3 & Total=21 \\
\hline
\end{tabular}

Now by applying the proposed Method, allocations are obtained as follows,

Total Cost obtained by proposed method is as follows,

Total Minimum Cost $=(2 * 4)+(6 * 3)+(1 * 7)+(1 * 2)+(3 * 1)+(6 * 4)+(2 * 16)$

$$
=94
$$




\section{Conclusion}

The main aim of this paper is to deriving the optimal transportation cost by using the above proposed method with less number of steps and it's very easy to understand. Based on the optimal solution it allows us to taking a decision effectively. Thus, there are possible extensions to improve our algorithm. The decision maker goes through all the steps of algorithm which makes our approach very useful to be applied in a lot of real world problems.

\section{Acknowledgements}

I would like to express my sincere gratitude to Dr. Bhavin. S.Patel, Assistant Professor, Applied Science and Humanities Department at Sankalchand Patel College of Engineering,Visnagar for his invaluable guidance, cooperation, constant encouragement, support, helpful comments, insightful suggestions and recommendations. I truly appreciate his esteemed guidance and encouragement, his knowledge and company at the time of crisis would be remembered lifelong.

\section{References}

[1]. Bazaraa M.S., Jarvis J.J., Sherali H.D., 1990. Linear Programming and Network Flows, John Weily, Second Edition, New York.

[2]. Lai Y.J., Hwang C.L., 1992. Mathematical Programming Methods and Applications, Springer, Berlin.

[3]. Swarup K., Gupta P.K., Mohan M., 2006. Operations Research, Sultan Chand and Sons, New Delhi.

[4]. Charnes, Cooper (1954). The Stepping-Stone method for explain linear programming. Calculation in transportation problems. Management Science 1(1)49-69

[5]. Dantzing G.B (1963).Linear Programming and Extensions, New Jersey: Princeton University Press.

[6]. Hitchcock FL (1941). The distribution of a product from several sources to Numerous Localities, Journal of Mathematical Physics $201941224-230$.

[7]. Koopmans TC (1947). Optimum Utilization of the Transportation system proceeding of the International statistical conference, Washington D.C.

[8]. A Charnes, W.W. Cooper and A. Henderson. An Introduction to Linear Programming, Wiley, New York, 1953.

[9]. Taha. H. A., Operation s Research -Introduction, Prentice Hall Of India (PVT), New Delhi, 2004.

[10]. Sharma J.K., Operations Research - Theory and Applications, Macmillan India (LTD), New Delhi, 2005.

[11]. Sudhakar V.J., Arunasankar .N., Karpagam .T., A New Approach for finding an optimal solution for Transportation problems, European Journal of Scientific Research ,ISSN 1450-216X Vol.68 No.2(2012), pp 254-257. 